\title{
Interview with Abel Laureate 2020 Hillel Furstenberg
} Bjørn Ian Dundas and Christian Skau

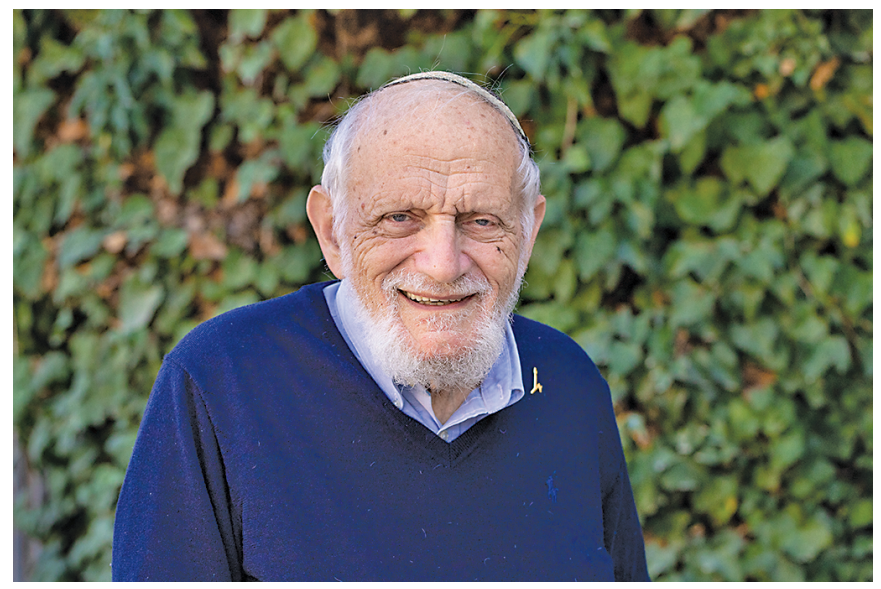

Hillel Furstenberg

Professor Furstenberg, first we would like to congratulate you on being awarded the Abel Prize in mathematics for 2020, which you share with Gregory Margulis. You have received the Prize, and here we cite the Abel Committee, "for pioneering the use of methods from probability and dynamics in group theory, number theory and combinatorics." Can you first of all tell us when you became enamoured with mathematics, and when you discovered that you had an exceptional talent for mathematics?

Bjørn Ian Dundas is a professor of mathematics at the University of Bergen. His research interests are within algebraic K-theory, homotopy type theory, and algebraic topology. His email address is dundas@math. uib. no.

Christian Skau is a professor emeritus of mathematics at the Norwegian University of Science and Technology (NTNU) at Trondheim. His email address is csk@math.ntnu.no.

This interview originally appeared in the December 2020 issue of the Newsletter of the European Mathematical Society and is reprinted here with permission of the EMS.

For permission to reprint this article, please contact: reprint-permission aams.org.

DOI: https://dx.doi.org/10.1090/noti2321
Perhaps I should say that I had a head start in mathematics-if you include adding and multiplying as mathematics. Let me give you the background story. I was born in Germany in 1935, and at roughly the age of five I came to the United States, my family having escaped Nazi Germany shortly after the Kristallnacht in November, 1938.

I lived with an uncle who had a poultry farm. I went to a rural school, which I think had only four classrooms. When I was in the kindergarten, I was in the same class as the first grade and second grade pupils. So it was easy for me to get a little bit ahead of where I should have been, or where I might have been. That was one aspect. Another aspect was that I had a sister who was three years older than I was, and she always kept me ahead. When the class was learning addition, I was learning multiplication. When they were learning fractions, I was learning algebra. I was always a little bit ahead, and of course you feel good about things that you are better at than most of the pupils in the class.

When I was in high school, I really enjoyed the Euclidean geometry that was taught there. I guess I enjoyed the challenge of geometry exercises. You are able to do things your way. You do not have to follow definite rules, it is about your own thinking. If it is clear and logical, you get to the right answer. I enjoyed that.

We learned about imaginary numbers when I was in high school. I thought I could make my name in mathematics if I proved that, when using imaginary numbers like $\sqrt{-1}$, it was going to lead to some contradiction in mathematics. I filled pages and pages of calculations and of course it didn't get anywhere, but it was a good experience just doing the calculations. I think it was a good experience to be a little frustrated when you want to show something, but it doesn't work out. But still, you don't feel bad about it. I think that it was pretty clear that I was enjoying mathematics. 
So you enjoyed problem solving?

Yes, absolutely. I should mention a friend of mine who became a prominent mathematician at Harvard-Shlomo Sternberg. He and I were in the same class at high school. Both of us heard about an interesting and challenging problem in Euclidean geometry: given a triangle, assume two of the angle bisectors have the same length. Prove that the triangle is isosceles. It is obviously trivial to do it the other way around. That is a rather difficult problem which I would have trouble doing today. Anyway, we came out each with our own solution to this after some time.

Did the two of you announce that you had solved this problem?

It so happened that the high school I went to was in the same building as Yeshiva College, and at that time there was a journal called Scripta Mathematica, which contained articles about recreational and historical aspects of mathematics. It was a good journal at that time, but it no longer exists. At any rate, the editor of that journal was Professor Jekuthiel Ginsburg, and he had an office in the same building as our high school.

Shlomo and I plucked up our courage and went up to Professor Ginsburg and showed him that we had solved this problem. He took it upon himself to encourage our interest in mathematics. In particular, he encouraged me further. He gave me opportunities to advance in mathematics and, at the same time, earn money. Our financial situation at home was not that good. My mother was widowed when we were on our way to the United States, and it was clear that I should be earning money to help out.

What Professor Ginsburg did was to give me a job with the journal. I would help out with the graphics, I would translate articles written in French and German to English. In many ways he encouraged me, and I owe a lot to him. He communicated to me and to his students the innate beauty of abstract mathematical ideas. Over and beyond the mathematics I learned, I experienced the love of mathematics blended with human kindness, an experience I can only wish I could replicate for others. Anyway, with this background I think it is pretty clear that mathematics was a direction that I wanted to go into.

While you were an undergraduate student at Yeshiva College you published three papers, two of which appeared in American Mathematical Monthly. We will focus on one of these papers titled "On the infinitude of primes." The paper is only half a page in length, but that belies its originality. Furthermore, perhaps this paper gave you the motivation to further explore the interplay between topology and dynamical systems on the one hand and number theory on the other hand? Could you elaborate on this?
Let me just say that I was recently asked what made me think about putting a topology on the integers. I didn't have the answer right away. Afterwards, I realised that at that time I had been learning about $p$-adic integers with the p-adic topology. So you could put a non-trivial topology on the integers, where integers which are normally very far away now are very close. For example, $n$ ! is very close to 0 , and so on. So I had this topology on the integers in which all non-empty open sets were infinite. By looking at it carefully you could prove that there must be infinitely many primes; otherwise one could show that there exists a non-empty finite open set.

Let me be more explicit. In our topology, two-sided infinite arithmetic progressions form the basis of the topology; so they play the same role as open subintervals of the unit interval $(0,1)$ in the usual topology. I hoped we could pursue the analogy further, and regard the set of integers also as a measure space with full measure 1 . Assuming the measure is translation invariant (as on the unit interval), an arithmetic progression $a+d \mathbb{Z}$ would have measure $1 / d$.

More generally, the measure of a "measurable" set would be its density. So this idea of looking at something happening in the integers as taking place in a measure space came in a natural way from the early paper, "On the infinitude of primes."

You graduated from Yeshiva College in 1955, having been awarded both a B.A. and an M.Sc. Then you went to Princeton University to study for your doctorate in mathematics, supervised by Salomon Bochner. Your PhD thesis, "Prediction Theory," was submitted in 1958, and in 1960 an elaboration of your thesis was published in Princeton Annals of Mathematical Studies under the title "Stationary processes and prediction theory."

What fascinated us greatly was that-as a by-product or offshoot of that work, really-you proved Hermann Weyl's celebrated equidistribution theorem by using dynamical systems. This was the first time that had ever been done, right?

As far as I know, yes. I didn't know of a precedent for that. Looking at number theoretic issues dynamically, I think that was a first.

\section{Could you elaborate?}

As you said, it came about in a rather indirect way, in studying prediction theory. First of all, the idea of looking at, say, the integers as a measure space leads to the next step of looking at it dynamically. In ergodic theory, one looks at a measure space with a transformation that preserves the measure. For the integers endowed with the density measure, translating a set by adding a constant also preserves the measure. So the idea of thinking of something happening on the integers as of dynamical nature is not unnatural. 
The motivation for me of working on prediction theory was related to my interest in harmonic analysis. Norbert Wiener, one of my mathematical heroes, had done some very profound work on Tauberian theorems in harmonic analysis. The latest thing that he had worked on was his prediction theory in a form closely related to harmonic analysis. In his theory, the "future," i.e., the next reading, was given as a random variable, that is, as a function defined almost everywhere on a measure space, not really well defined at specific points.

And so the question that arose, and that I wanted to answer, was: Suppose you're given exactly a certain past, past meaning something that happened yesterday, the day before, and so on up to minus infinity, so to speak. This I called the past. Given the past you would like to say exactly what's in the future, but you usually cannot say that. If, for example, what you are looking at is coin tossing, then all you could say is that with probability one half the next reading would be heads, and with probability one half it would be tails. So, going into the entire future, what you want to define is a stochastic process that will answer: What are the probabilities of what's happening in the future given what you have had in the past. There are certain situations in which you can do this. That's what is elaborated in my thesis.

\section{How does the ergodic theorem enter the picture?}

In fact, what I was doing was inverting the ergodic theorem. Let me explain. The first step in my construction, and this is how prediction theory connects with stationary processes, is to look at the past and to associate with it a stationary process. In other words, you want to look at this as a typical sequence of some stationary process. A stationary process arises by evaluating the function at hand on a probability space on which there is a measure-preserving transformation, the latter representing change in time. So the statistics of the process today and tomorrow are the same as the statistics will be a week from now and the day after, and so on.

The point is that I found a method of going from the individual sequence to the process, which is inverting the ergodic theorem. In fact, if you apply the ergodic theorem to stationary processes you can say in terms of expectations what is happening at almost every sample sequence. Our idea was to go from what I would like to be a sample sequence to the process of which it could be looked at as a typical sequence. This represents a reversed point of view. The idea is, you assume densities are defined in the past; let's say it's a plus one, minus one sequence. You notice that plus ones occur, say, 2 / 3 of the time in the past, and minus ones occur $1 / 3$ of the time, and they occur together $1 / 7$ of the time, and so on; for every combination there is a well-defined frequency of occurrence. Knowing this you infer the statistics from the given sequence, and from this statistics you build the stationary process.

\section{Are there alternative ways of looking at this?}

Yes, there is a more constructive way of building the space on which the stationary process is defined. In fact, just take the sequence itself and look at all its translates, and then take the closure in the product, or Tychonoff, topology. That gives you a compact space. On that space you define a measure based on what is "happening," so to speak, on the sequence you started with.

I mention this way of doing things because this has a precedent in Bochner's approach to almost periodic sequences, or more generally, to almost periodic functions. What he did was to say that almost periodic functions have a certain property: if you look at the closure of the translates of one such function, then you get a compact space in the strong topology. That it is compact in the weak topology is immediate, but that it is also compact in the strong topology turns out to characterise almost periodic functions. So this idea of embedding a single sample sequence in a whole family of sequences is due to Bochner, although I didn't know it at the time I was his graduate student.

Let's go back to your thesis where you, as we mentioned already, were able to prove Hermann Weyl's equidistribtion theorem by using dynamical arguments. We understand that this came as an offshoot, so to speak, of the main thrust of your thesis. At any rate, did this inspire you to look at other Diophantine approximation problems to see if you could solve these by using dynamics?

Yes, it did encourage me to look for dynamical systems relevant for other number theoretic problems. You may ask: How did my prediction theory lead me to such dynamical systems?

The basic idea is a little bit subtle and somewhat technical. For us, to predict is, given the past reading through yesterday, to produce a stochastic process, indexed by time $0,1,2$, etc., representing in probabilistic terms what will happen today and in the future. Moving ahead one day in time, we want to produce another stochastic process, also knowing today's reading. The new "future stochastic process" is just the old one, conditioned on today's reading and re-indexing $1,2,3$, etc. to 0,1 , 2, etc. So, if a point in our probability space comprises a pair (two-sided, past + future actualised sequence, future process) our dynamic transformation takes this point to the pair (shifted sequence, future process conditioned on 0-reading). Dynamically this is a complex example of a skew product dynamical system.

Can you give us an example of a skew product? 
Our space is a two-torus (the surface of a doughnut) which is formed by moving a vertical circle along a circular path returning to its initial position. We think of this as a bundle of circles above a base circle. A classic example named for Anzai and Kakutani describes a transformation of this torus whereby the base circle is rotated by a fixed angle, and the vertical circles move accordingly, each one rotated by an angle depending on its location on the base circle. For the example of Anzai-Kakutani you can show, using ideas from ergodic theory, that every orbit is equidistributed when the base circle is rotated by an angle $\alpha$, an irrational multiple of $\pi$.

This is interesting. It gives you the equidistribution of $n^{2} \alpha(\bmod 1), n \in \mathbb{Z}$, originally proved by Weyl by his methods of trigonometric series. So this gives a dynamic proof of Weyl's theorem. That encouraged me to look in general at a sequence as a sample sequence of some dynamical system, and then study the dynamical system and see what you can say.

Is it fair to say that this way of thinking gave you the idea of how to prove the Szemerédi theorem, which says that a subset of the integers with positive upper density has arithmetic progressions of arbitrary length?

Yes, you are basically correct. Let me put it this way: looking at the integers as a measure space, where adding one to each integer is a measure-preserving transformation, we're given a set of positive measure, and I want to show that I can return several times to that set using the same number of steps. That I return once-in the standard probability space context-is the Poincare recurrence theorem, but what I want is what is now called multiple recurrence. Given any natural number $n$, there exists a number $m$ such that I return to the given set $n$ times using consecutively $m$ steps. This phenomenon of repeated recurrence is the measure theoretic version of the Szemerédi theorem.

This is a prime example of what is now called the correspondence principle. Something going on in the integers corresponds to something going on in a measure space. You prove the measure theoretic thing, and then you get the number theoretic thing. In my thesis this principle does not appear explicitly, but it is implicit. The idea of how to go from an explicit past to a process is basically the correspondence principle. That's really the first time that the principle was used in a way that could be called a principle.

It is noteworthy that Green and Tao in their proof of the celebrated result that the primes contain arithmetic progressions of arbitrary length, while making no explicit use of ergodic theory, are influenced in their approach by the novel methods you apply in proving Szemerédi's theorem, specifically your correspondence principle.
In 1981 you published a book titled Recurrence in Ergodic Theory and Combinatorial Number Theory. It is a marvellous book which has enthralled many, including the two of us. It describes in exemplary clarity how one can apply dynamical systems-both topological and ergodic-to number theory and Diophantine approximations, thereby proving some highly non-trivial results.

We will return to your proof of the multiple recurrence theorem, but first it might be useful that you tell us what an isometric extension is, and how this concept is the key building block in your proof from 1963 of the structure theorem of so-called distal flows in topological dynamics.

The Anzai-Kakutani example that I mentioned earlier will give a good illustration of what an isometric extension is. So, you have a big topological dynamical system on the two-torus, and a smaller one on the base circle. We say that the big system is an extension of the smaller one in the sense that looking at two coordinates $(x, y)$ describing the torus, you now look at the first coordinate $x$ which parametrises the base circle. That $x$ coordinate moves according to a certain rule; in our case, $x$ goes to $x+a$, and the $y$ goes to some other $y$. So sending $(x, y)$ to $x$ is a factoring of the big system to a smaller system. But it is factoring in a special way, which is an example of an isometric extension, in the sense that two points on the torus that sit over the same point of the base circle maintain a fixed distance from each other as the $x$ coordinate moves. The big system is an example of a so-called distal system, meaning that if two points are distinct they don't get closer than a certain amount, which depends upon where the points are.

Obviously, if a dynamical system is isometric, meaning that distances are preserved under the dynamics-we assume the underlying space is a compact metric space-then the system is distal. For a time it was an open question if distality implies isometry, perhaps in a different, but compatible, metric. This is in fact correct if the space is zero-dimensional-in particular, if the space is the Cantor set-and was proved by Robert Ellis in 1958. However, one can show that the Anzai-Kakutani example is distal, but not isometric, so the converse is not true. What you can show is that an isometric extension of a distal system is again distal.

So, to get examples of distal systems you take successive isometric extensions, even infinitely many, of a given distal system. These were the only examples of distal systems that I knew about, so I asked myself: Maybe that's it, there are no other examples? I proved that fact, and that became the structure theorem for distal systems.

Is there an analogous structure theorem for ergodic systems in the measure theoretic setting?

That's the crucial point in the proof of the ergodic version of the Szemerédi theorem! Firstly, you have something 
analogous to what I described in the topological setting, which you could call a distal ergodic system. Every ergodic system has as its base a distal factor, which might just be a rotation. But that does not necessarily exhaust the whole system, because an ergodic system is not in general distal. The next-and final-step to get the ergodic system you are looking at is a so-called relatively weak mixing extension. The notion of weak mixing means that things get very mixed up, and there is a relative notion of that. So the most general ergodic system is obtained by a relative weak mixing extension of a distal system.

What is that good for? Well, in this way I can prove the Szemerédi theorem in its ergodic version using that structure, by proving it bit by bit: proving that it's true for distal systems, which means it's true for rotations and isometric extensions, and then showing that if it's true for a given system, then it's true for a weak mixing extension of that system.

Could you tell us how you became aware of the Szemerédi theorem in the first place, as well as the origin of your proof of that theorem?

That was sort of accidental. The year 1975 was the first year of the Institute for Advanced Studies at the Hebrew University in Jerusalem, and the application by the Mathematics Department to have a special year devoted to ergodic theory was accepted and funded. We invited ergodic theorists from around the world to attend, like Donald Ornstein, Daniel Rudolph, Jean-Paul Thouvenot, and many others. Then there was Konrad Jacobs, who was one of the early authors of a book on ergodic theory. But at that time he had stopped being interested in ergodic theory and had become interested in combinatorics instead, and so was not a member of our ergodic theory group.

Anyway, Jacobs was invited to visit, and he suggested to the organisers to give a colloquium talk on some aspects of combinatorics that he found exciting, including, as it turned out, the Szemerédi theorem. I was really not interested in the topic of his talk, but I felt that out of respect for the speaker, I should attend the lecture.

At that time I was basically aware of the correspondence principle that we talked about, so hearing of Szemerédi's theorem from Jacobs, it was natural to translate it into ergodic theoretic terms. Also, at that time I had information about what you can say about weakly mixing systems. It turns out that for weakly mixing systems, you have recurrence more or less in any pattern you want. In particular, you have recurrence along an arithmetic progression. So if the system is weakly mixing, you are finished. The other extreme case relative to weak mixing is rotation on a compact group. Again, it's almost immediate that recurrence occurs along arithmetic progressions. What was needed to nail the proof was a structure theorem, combining the two modes of behaviour. With the help of colleagues, Benjamin Weiss and Yitzhak Katznelson, I succeeded in proving the necessary structure theorem.

The story you have just told us seems to be an example of cross-fertilisation between different mathematical perspectives. Going to department colloquiums, even though the topic is vastly different from one's own interests, can open one's eyes to see what one can do.

Oh, yes, absolutely. I certainly learned a lesson from that. Actually, at my retirement there was a conference held in Jerusalem, and I was asked to speak on "Probability in Mathematics." I was thinking about my own career, and it struck me that I should really call my talk "The improbability of my mathematics." There were so many things that came together in my mathematical life. For instance, had I not looked at distal systems, I wouldn't have known how an appropriate structure theorem for ergodic systems might be formulated.

Incidentally, there was another accident that I should mention. I was in fact not going to be able to go to the colloquium talk by Konrad Jacobs. My youngest son had just been born a few months earlier, and at the time of the colloquium, I was to be assigned babysitter. Fortunately, my eldest child, my daughter, happened to be free at that time, and she came and took my place. Had she not, I would not have heard this colloquium talk.

Before we leave the subject of your ergodic proof of the Szemerédi theorem, we should emphasise that this has spawned a lot of generalisations due to many people, including you and various co-authors. One of the most spectacular of these generalisations is your and Katznelson's proof in 1991 of the density version of the Hales-Jewett theorem, which is a fundamental result in Ramsey theory. The proof is achieved by means of a significant extension of the ergodic techniques that you had pioneered in your proof of the Szemerédi theorem, and the result did not seem to be available by any other means than ergodic theory. However, in 2012 a so-called Polymath group of mathematicians published a new proof avoiding ergodic theory arguments. That is, they do admit that some part of their proof is inspired by ergodic methods. Do you have any comments?

It wasn't unnatural that one would find a combinatorial proof for a combinatorial theorem. But I think that in every proof (as with Gowers' proof of the original Szemerédi theorem), one decomposes the behaviour to a random component and a regular component.

You introduced another concept which has been immensely important, namely that of a boundary. But perhaps before we get there, could you say something about random walks and how it is related to the boundary concept? 
First let me give you an example of a random walk in a group. Say I am given a bunch of $m \times m$ matrices and I attach a probability for each of those. I decide to start multiplying these matrices randomly according to that probability distribution. So I get the matrices $X_{1}, X_{1} X_{2}, X_{1} X_{2} X_{3}$, and so on, and it turns out that it is, with some restrictions, rather easy to show that the norms of this sequence grow exponentially. But I want to know what is happening qualitatively. What I am looking at is a random walk inside a group of matrices and I want to look at some limiting behaviour. It's no longer true that with probability one there is a specific limiting behaviour.

What behaviour is there then that can be called upon? It turns out that the rows of these product matrices come closer and closer together as you go to infinity, and they tend to point in a certain direction. A different sequence of products of matrices would give you a different direction, so you wind up with a random direction in projective space, basically. If you look at the special case of $2 \times 2$-matrices this is the only kind of boundary behaviour that you can talk about, in the sense that you can ask which point on the projective line does the sequence of products converge to.

In higher dimension it turns out that the sequence of products of matrices converges to a point in a so-called flag space of the right dimension-a line sitting in a plane, a plane sitting in a 3-space, and so on. In some sense, I can attach a flag space of dimension $m-1$ to the group $\mathrm{GL}_{m}(\mathbb{R})$ of $m \times m$ invertible matrices. So it makes sense to talk about a random walk converging to a point. What is nice about this is that for many groups (e.g., semi-simple Lie groups), this boundary has an explicit presentation as a homogeneous space of the group.

This is what is called the Furstenberg boundary today? Can this boundary be characterised in another way?

Yes, it can be characterised abstractly in terms of the notion of strong proximality. Proximality means that it is opposite to distality-the notion we already have encountered. So, we have a compact space on which the group acts in such a way that any two points will get as close together as you like under the action of some group element. If you think about it, that entails that any $k$ points can come close together under the action of a group element.

Strong proximality means that if you have any measure on the compact space on which the group acts, it will converge to a point measure-a Dirac measure-under the action of some sequence of group elements. It's not obvious that the two notions are different, but they are. Now the boundary of the group can be characterised as the universal strongly proximal action of the group.

Margulis, with whom you share the 2020 Abel Prize, writes somewhere, and we quote: "I learned about Furstenberg's work around 1974, and his boundary theory influenced me very much. In particular, my proof of the normal subgroup theorem concerning lattices in semi-simple Lie groups could not exist without that theory. I also consider the proof of the normal subgroup theorem as my best proof." So he credits you for supplying him with a crucial idea in his proof!

I am very happy to hear that! I might actually give someone else credit here, namely the probabilist Monroe Donsker, who put me in contact with a friend of his-his name was Peter Ney-who was editing a book on applications of probability in various mathematical fields. He came to me once when I visited the University of Minnesota and said: "You should find an application of probability to algebra for Ney's book." So I thought of the boundary theory, and I thought that it seems intuitive that a lattice subgroup of a group should be very close qualitatively to the group itself. In fact, these two ought to have the same boundary.

Using this idea, I could prove a very special case of a theorem of Margulis-his superrigidity theorem. So in that way I interacted with Margulis. That you could use the boundary to reflect, so to speak, properties of the group itself turned out to be useful.

You mentioned to us before that Gelfand's work on Banach algebras, and, in particular, on commutative $C^{*}$-algebras, was an important inspiration for you at some point. Could you elaborate on that?

There are two ways in which Gelfand's theory comes in. One way of proving the correspondence principle is by using Gelfand's proof that there exists an isometric isomorphism between an arbitrary commutative $C^{*}$-algebra and an algebra of continuous functions on a certain compact space, sometimes called the Gelfand space. Applying this to the algebra of bounded sequences produces the space in which the dynamics takes place.

In fact-it is sort of an anecdote but this really happened-I once gave a lecture on these things at Gelfand's seminar at Rutgers University. Gelfand liked, when you put forth a theorem, to understand that theorem by himself. He did not want to listen to the lecturer explaining the theorem. I had put the correspondence theorem on the blackboard: so given something on the integers, then there's a measure space, etc., etc. Gelfand turned to the class in the seminar and asked: "Why is this true?" He didn't know either, but he wanted someone to explain it. Everybody gave up. And then I said: "It's Gelfand's $C^{*}$-representation theorem; that's how you get this."

The other thing I would mention that what Gelfandwho certainly was one of my mathematical heroes-did, was to algebraicise and then prove the Wiener Tauberian theorem. This was one of the things I learned when I was a student at Princeton, and it made a deep impression on 
me. Gelfand gave this new and marvellous proof invoking algebra, and, of course, at a certain point you need analysis. Now one might say that Gelfand's theory is also the basis of my original way of getting the boundary of a group. In fact, there is a connection between boundaries and harmonic functions, and you somehow build an algebra from harmonic functions by finding a certain way of multiplying these functions. The Gelfand space of that algebra is the boundary of the group.

Of course, what is important here is the connection between random walks and harmonic functions. In fact, you can look at harmonic functions by looking at probabilistic questions on random walks, and vice versa, you can go from harmonic functions to random walks.

In 1967 you introduced the notion of disjointness of ergodic as well as topological dynamical systems. This notion, which is akin to that of being coprime for integers, turned out to have applications to a wide range of areas, including signal processing and filtering questions in electrical engineering, the geometry of fractal sets, homogeneous flows and number theory. Could you comment on this?

Let me answer your question in the following way. The notion of disjointness in dynamical systems arises in connection with filtering. Specifically, filtering out noise from a signal consisting of a transmitted time series plus noise. Given that signal and noise have known stationary statistical behaviour, when can noise be filtered out entirely? A sufficient condition is "disjointness" of the underlying dynamical systems generating the signal and the noise. This notion, while originating in the ergodic context, also applies to topological dynamical systems and gives insight into the structure of various systems.

There is an incidental application to Diophantine approximation involving the dynamics of two transformations acting on a space; for example $x \rightarrow 2 x(\bmod 1)$ and $x \rightarrow 3 x(\bmod 1)$, where 2 and 3 are examples of "multiplicatively independent" integers. The theorem states that when both operations are applied-so higher rank actions-then the orbits are either finite or dense.

The underlying intuition was that the two actions are fundamentally distinct, so that the invariant sets of each will also be different, so that a common invariant set would necessarily be degenerate-either finite or the whole space. My 1970 paper titled "Intersections of Cantor sets and transversality of semigroups" is an effort to make this precise. In differential and algebraic geometry, the notion of transversality relates to the dimension of the intersection of the two manifolds. In our context, Hausdorff dimension is expected to play a similar role, and a number of conjectures are raised in the paper. A partial result is obtained for which the underlying idea is the construction-based on a given fractal measure-of a stationary process of measure-valued random variables.

You wrote a memoir titled Ergodic Theory and Fractal Geometry in 2014, where you try to reignite interest in this subject. Can we ask you what the status of the conjectures you alluded to above is?

Very recently one of my main conjectures was proved by two mathematicians, Meng Wu and, independently, Pablo Shmerkin. To illustrate the result they proved one has the following corollary: the inequality

$$
\begin{aligned}
\operatorname{dim}\left\{2^{n} \alpha(\bmod 1) \mid n \in \mathbb{Z}\right\} & \\
& +\operatorname{dim}\left\{3^{n} \alpha(\bmod 1) \mid n \in \mathbb{Z}\right\} \geq 1
\end{aligned}
$$

holds for all $\alpha$ in $\mathbb{R}$ except for a set of Hausdorff dimension 0 . Here "dim" is box dimension, which is Hausdorff dimension of closure. Obviously, if $\alpha$ is rational, then it is in the exceptional set. The details of the use of ergodic theory in the context of fractals and their dimensions appear in the monograph you referred to.

At any rate, there is current interest in that aspect, that is, the connection between ergodic theory and fractal geometry. Actually, Wu's proof makes use of the ergodic theory of something called a CP process, which comes about naturally when you try to look at things as a kind of process. You're zooming in at a fixed rate. Now, what are you seeing on your screen? How does this picture change? Instead of numbers changing, there are pictures changing. And that could all be a stationary process. Every fractal generates some kind of stationary process which is interesting to look at. I am very happy about the current interest in all of this.

As we emphasised at the outset of this interview, you were the first to build a bridge between dynamical systems and number theory, in particular, combinatorial number theory and Diophantine approximations. This has spawned a lot of activity in this area, both among your colleagues and your students, and again their students. You must be very pleased by this development?

Certainly. One is proud of one's children, but maybe in a sense even more proud of the grandchildren. It shows there's a line going there. The same is true in mathematics: what your students can do, and what their students are doing, likewise with your colleagues, enables you to see and appreciate the ramifications of your work. The real prize, and what you really appreciate, is when people understand what you are doing and are continuing that.

We still have bunches of other questions on our notepads, but perhaps this is the time we should end this interview. We would like to end by quoting Harish-Chandra: "I have often pondered over the roles of knowledge or experience, on the one hand, and 


\title{
COMMUNICATION
}

imagination or intuition, on the other, in the process of discovery. I believe that there is a certain fundamental conflict between the two, and knowledge, by advocating caution, tends to inhibit the flight of imagination. Therefore, a certain naiveté, unburdened by conventional wisdom, can sometimes be a positive asset."

It seems to us that you in your approach to mathematics embody what Harish-Chandra describes.

I'm happy if you think of it this way!

On behalf of the Norwegian and the European Math. Societies, and from the two of us personally, we would like to thank you for this most interesting interview. We very much look forward to meeting you in person in Oslo at the next Abel Prize event. ${ }^{1}$

I am looking forward to that myself, among other reasons in order to put things on the blackboard so that people will really be able to understand the things we have been talking about abstractly.

${ }^{1}$ Due to the COVID-19 pandemic, the prize ceremony for the Abel Prize 2020 had to be postponed. The interview was conducted remotely, with Professor Furstenberg at home in Israel.



Bjørn lan Dundas

Credits

Photo of Hillel Furstenberg is courtesy of Yosef Adest/Hebrew University of Jerusalem.

Photo of Bjørn Ian Dundas is courtesy of Halvard Fausk. Photo of Christian Skau is courtesy of Christian Skau.


\section{MemberGetAMember Program}

\author{
When your friend joins or renews their AMS membership, \\ you each receive 25 AMS points! \\ Start now: www.ams.org/member-get-a-member
}

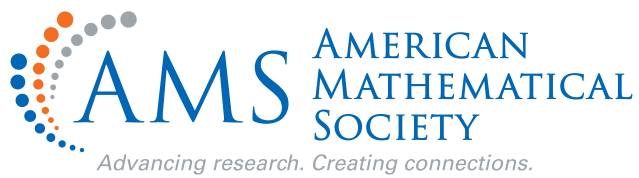

* Affiliate, Emeritus, and Nominee members are not eligible for this benefit. 